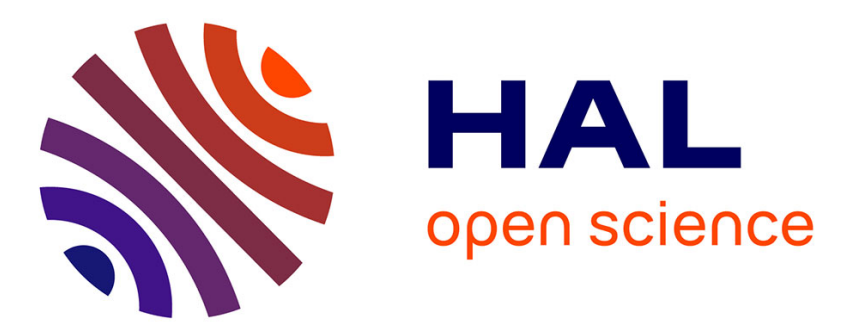

\title{
Origine et principaux caractères des souches françaises d'Hypoxylon mammatum (Wahl.) Miller \\ Jean Pinon
}

\section{To cite this version:}

Jean Pinon. Origine et principaux caractères des souches françaises d'Hypoxylon mammatum (Wahl.) Miller. European Journal of Forest Pathology, 1979, 9 (3-4), pp.129-142. 10.1111/j.14390329.1979.tb00672.x . hal-02731288

\section{HAL Id: hal-02731288 \\ https://hal.inrae.fr/hal-02731288}

Submitted on 2 Jun 2020

HAL is a multi-disciplinary open access archive for the deposit and dissemination of scientific research documents, whether they are published or not. The documents may come from teaching and research institutions in France or abroad, or from public or private research centers.
L'archive ouverte pluridisciplinaire HAL, est destinée au dépôt et à la diffusion de documents scientifiques de niveau recherche, publiés ou non, émanant des établissements d'enseignement et de recherche français ou étrangers, des laboratoires publics ou privés.

\section{다(1)(2)}

Distributed under a Creative Commons Attribution - ShareAlikel 4.0 International 


\title{
Origine et principaux caractères des souches françaises d'Hypoxylon mammatum (Wahl.) Miller
}

\author{
Par J. PinON
}

Réception du Ms.9.6.1978

\begin{abstract}
The origin and the main characters of French isolates of Hypoxylon mammatum (Wahl.) Miller. The geographical distribution and the historical origin of aspen canker in France are described and discussed.

Isolates are variable in both qualitative (pigmentation, sporulation) and quantitative (temperature requirements, growth) characters. The fungus is able to decompose wood and shows celluly tic activity in vitro.
\end{abstract}

\section{Introduction}

Le chancre des trembles à Hypoxylon mammatum (Wahl.) Miller a longtemps été considéré comme une maladie inféodée à l'Amérique du Nord. Cette conception n'est plus valable du fait des mentions européennes dont il a été récemment l'objet. Kalandra (1966) puis Urosevic (1972) l'ont décrit en Tchécoslovaquie. De nombreuses régions de l'URSS l'hébergent actuellement: Moscou, Koursk, Kouibychev, Voronej, Tambov, Rostov, l'Oural central et méridional, Novosibirsk (Shirniva et Kireeva 1976). Nous l'avons signalé en France et en Italie (Pinon 1975). Des prospections en Europe septentrionale et centrale (surtout en zone alpine) devraient conduire à une meilleure connaissance de la répartition européenne du parasite.

Son seul hôte indigène en France est le tremble (Populus tremula L.), essence marginale et relativement négligée sur le plan forestier. Toutefois, un travail important de sélection a été mené depuis une vingtaine d'années sur la section Leuce du genre Populus (Lemoine 1973). Des familles d'hybrides interspécifiques de bonne vigueur et bien adaptés aux sols hydromorphes (qui excluent la populiculture classique) ont été obtenues. Il s'agit principalement d'hybrides $P$. alba $\times P$. tremula et $P$. tremula $\times P$. tremuloides (ou $P$. tremula $\times P$. grandidentata). Avant d'entreprendre la diffusion et la culture de ces végétaux, et en particulier de ceux qui comportent P. tremuloides pour parent mâle, il convient de s'assurer de leur tolérance au chancre. La présente étude constitue une étape préliminaire qui doit mener ultérieurement à la mise au point d'un test de sensibilité et à une sélection sanitaire.

\section{Répartition d'Hypoxylon mammatum en France}

En 1975, les foyers connus d'infection par H. mammatum relevaient de deux types de situation. Tout d'abord des foyers alpins, sur trembles sauvages de la race montagnards, et considérés comme spontanés. D’autre part, des arbres infectés dans deux parcelles expérimentales de l'Est de la France (Nancy et Epinal) dont l'origine des plants était commune (la pépinière de Nancy). Les trembles sauvages situés dans les massifs forestiers entourant ces 
dernières parcelles ont été alors examinés, sans que nous puissions y déceler la présence de chancre.

Depuis, nous avons poursuivi notre prospection et la fig. 1 récapitule les foyers actuellement connus. Non seulement de nouveaux foyers alpins ont été repérés, mais aussi des cas

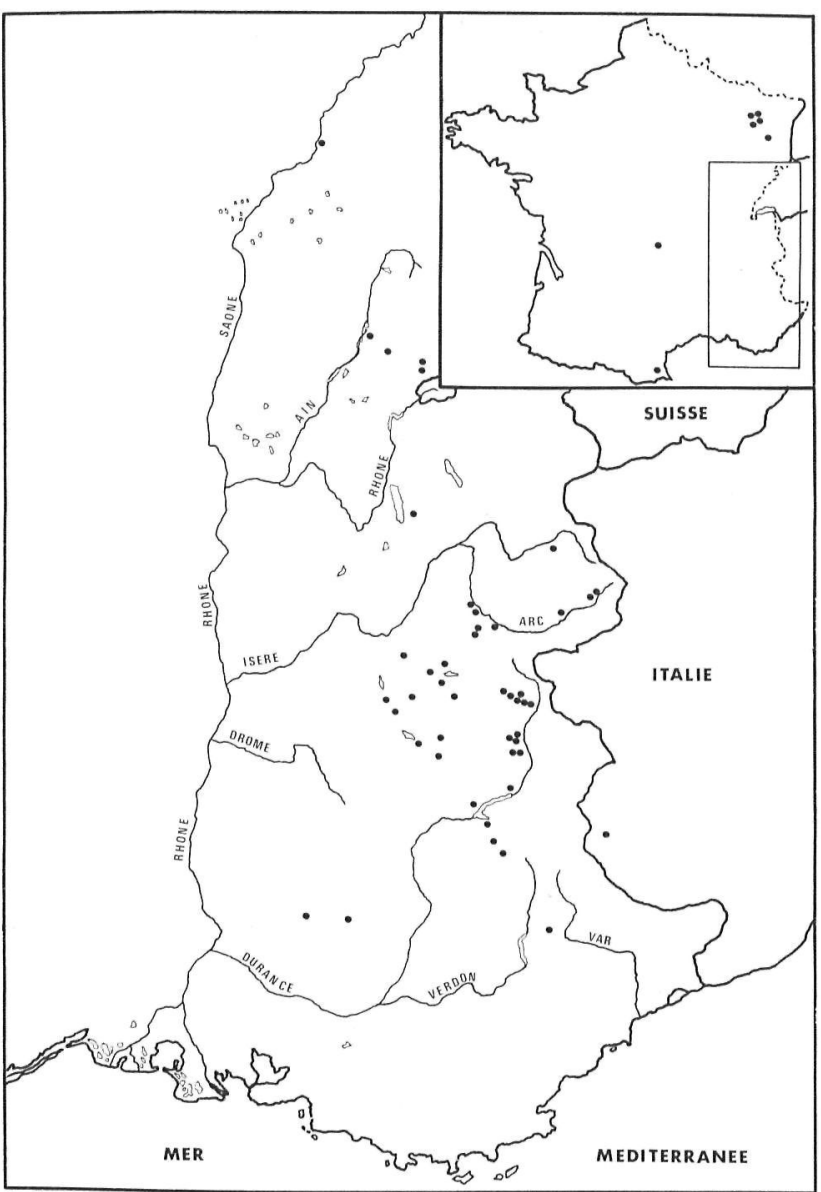

Fig. 1. Répartition géographique des foyers d'H. mammatum repérés à ce jour. En encadré : la région Rhône-Alpes nous ont été signalés en Bourgogne et dans le MassifCentral (communication de C. Delatour), dans le Jura et en Lorraine (communication de R. Perrin). Enfin, nous avons relevé un cas dans les Pyrénées.

A présent, les foyers peuvent schématiquement être classés en deux catégories que nous nous proposons de décrire ci-dessous.

\section{Caractères des peuplements infectés}

Lorsque les trembles appartiennent à la race de plaine, telle que décrite par Silvyy-Leligeois (1949), c'est-à-dire à écorce grise, rhytidome précoce etforme défectueuse, le taux d'infection est extrêmement faible. Ces trembles croissent en mélange avec d'autres feuillus (chênes, chataigniers, hêtres) sur sols bruns, souvent hydromorphes. Ils ne forment qu'exceptionnellement des peuplements importants et leur dispersion n'est guère favorable au parasite. Les infections connues le sont le plus souvent en lisière des massifs forestiers. Ces trembles se rencontrent en plaine et à faible altitude en montagne sur des terrasses ou des fonds mouilleux. Si l'infection peut conduire à la mort de l'arbre, en fait, il apparaît souvent des bourrelets cicatriciels, insuffisants pour limiter l'extension du parasite. Généralement, la cime casse ou dépérit.

Le second cas est spécifique des trembles de la race montagnarde. Nous les avons rencontrés à toutes altitudes (de 200 à $1850 \mathrm{~m}$ ) dans les Alpes et aussi dans le Sud du Jura. Les peuplements y sont purs, rarement très importants, pouvant parfois s'étendre sur près d'un hectare, fréquemment colonisant des prés abandonnés. Ils croissent sur des pentes, sur des sols variés souvent superficiels et alimentés en eau par de fins ruisseaux. Droits, leur écorce est de couleur claire (jaune à verdâtre), et leur rhytidome tardif. Fréquents dans le Brian- 
çonnais, ils colonisent l'étage montagnard et parfois le subalpin. Ici, la plupart des peuplements visités a révélé des infections, dont le taux est de l'ordre de $1 \%$ des sujets mais peut atteindre 10\% et exceptionnellement plus sur le Plateau d'Albion et dans la région de Seyne (Alpes du Sud). L'infection des jeunes sujets évolue rapidement et les tentatives de cicatrisation sont aussi rares qu'incomplètes. Le parasite y paraît nettement plus actif. Il est évident que des hybrides peuvent exister, en particulier dans les Alpes, entre les deux races de Tremble et que leur identification est alors difficile.

Pour rendre compte de ces comportements différents du couple hôte-parasite, on doit à priori envisager trois hypothèses : effet génétique (hôte), effet du milieu (station) ou variabilité du parasite. C'est sur ce dernier point que porte l'expérimentation que nous relaterons par la suite. Mais avant, nous tenterons de définir l'origine la plus probable de la maladie en France et même en Europe.

\section{Hypothèses sur l'origine de la maladie}

Il est maintenant acquis que le parasite sévit de longue date en France. Ainsi, dans les Alpes, les foyers sont séparés par de hautes chaines montagneuses, sans continuité de la végétation

\section{Tablean 1}

Hypoxylon mammatum : espèces réunies en synonymie par MILLER

\begin{tabular}{|c|c|c|c|}
\hline $\begin{array}{l}\text { Espèce et référence bibliographique } \\
\text { (in MILLER p. 64) }\end{array}$ & lieu de récolte ${ }^{2}$ & Hôte primaire ${ }^{2}$ & $\begin{array}{l}\text { Hôtes ajoutés } \\
\text { in Oudemans }\end{array}$ \\
\hline $\begin{array}{l}\text { Sphaeria mammata Wahl., Flora } \\
\text { Suecica, p. } 1003,1826\end{array}$ & Amérique du Nord & $\begin{array}{l}\text { Salix caprea } \\
\text { Betula alba }\end{array}$ & \\
\hline $\begin{array}{l}\text { S. mammiformis Wahl., Flora lappo- } \\
\text { nica, p. } 520,1812\end{array}$ & Laponie & $\begin{array}{l}\text { Salix caprea } \\
\text { Betula crassiori }\end{array}$ & \\
\hline \multicolumn{4}{|l|}{$\begin{array}{l}\text { S. multiformis var. B., Fr., Elench. } \\
\text { Fung., p. } 64,1828{ }^{1}\end{array}$} \\
\hline $\begin{array}{l}\text { S.pruinata Klotz, Linnea S, p. } 489 \text {, } \\
1833\end{array}$ & Amérique du Nord & P. tremula & \\
\hline Hypoxylon karstenii Fr., nom. nud. ${ }^{1}$ & & Salix & \\
\hline $\begin{array}{l}\text { H. pauperatum Karst., Not. ur Sällsk. } \\
\text { pro Fauna et Flora Fenn. Förh., I, } \\
\text { p. } 211,1866\end{array}$ & Laponnie & $\begin{array}{l}\text { Salix phyllicifo- } \\
\text { lia, S. nigricans }\end{array}$ & $\begin{array}{l}\text { P. tremula, } \\
\text { Sorbus aucuparia }\end{array}$ \\
\hline $\begin{array}{l}\text { H. macrosporum Karst., Myc. Fenn. 2, } \\
\text { p. } 40,1873\end{array}$ & Laponie & Salix & \\
\hline $\begin{array}{l}\text { H. morsei Berk. \& Curt., Grevillea 4, } \\
\text { p. } 51,1875\end{array}$ & Etats-Unis & Alnus & Pirus malus \\
\hline $\begin{array}{l}\text { H. blackei Berk. \& Curt., Grevillea } 4 \text {, } \\
\text { p. } 52,1875\end{array}$ & Etats-Unis & Salix & \\
\hline $\begin{array}{l}\text { Rosellinia pruinata (Klotz.) Sacc., } \\
\text { Syll. F. 1, p. 259, } 1882\end{array}$ & Cf. S.pruinata & Cf. S.pruinata & \\
\hline $\begin{array}{l}\text { H.pruinatum (Klotz.) Cke., } \\
\text { Grevillea 11, p. 127, } 1883\end{array}$ & Cf. S.pruinata & Cf. S.pruinata & \\
\hline $\begin{array}{l}\text { H. bolwayi Ell., Amer. Nat., 17, } \\
\text { p. } 193,1883\end{array}$ & Etats-Unis & Populus & P. tremula \\
\hline $\begin{array}{l}\text { Anthostoma morsei (Berk. \& Curt.) } \\
\text { Cke, Grevillea 11, p. 127, } 1883\end{array}$ & Cf. H.morsei & Cf. H.morsei & \\
\hline $\begin{array}{l}\text { Fuckelia morsei (Berk. \& Curt.) Cke, } \\
\text { Grevillea } 12 \text {, p. } 52,1883\end{array}$ & Cf. H.morsei & Cf. H.morsei & \\
\hline H. cameratum Ell. et Holw., nom. nud. & Amérique du Nord & Betula & \\
\hline $\begin{array}{l}1 \text { espèce dont nous n'avons pu consulter } \\
2 \text { données figurant dans la description or } \\
\end{array}$ & $\begin{array}{l}\text { description originale } \\
\text { inale }\end{array}$ & & \\
\hline
\end{tabular}


ligneuse. Messiaen, qui nous a procuré des échantillons du Plateau d'Albion, nous a déclaré qu'il connaissait ce faciès sur tremble depuis une vingtaine d'années. Bouvarel et Fourchy (1954) ont décrit dans l'Oisans un dépérissement qui, à posteriori, pourrait bien être celui provoqué par H. mammatum.

En plaine, la dispersion géographique des foyers, alors que l'inoculum y est infime, atteste aussi du caractère ancien de la maladie. Celle-ci est en fait passée longtemps inaperçue, le tremble n'étant guère étudié par les pathologistes.

Rien ne permet de croire à une introduction de la maladie d'Amérique du Nord: il est douteux que des échanges commerciaux aient porté sur le Tremble et le matériel introduit pour la sélection était en fait constitué de pollens. Les autres mentions européennes de la maladie (URSS et Tchécoslovaquie) semblent tout aussi incompatibles avec l'hypothèse d'une introduction du parasite d'Amérique du Nord. Elles confortent notre point de vue sur l'ancienneté de la maladie sur notre continent.

Faute de pouvoir retracer l'histoire du parasite, nous nous sommes penchés sur la bibliographie et plus particulièrement celle ayant trait à la systématique. Miller (1961) a proposé pour cause d'antériorité le binome $H$. mammatum, réduisant en synonyme de nombreuses espèces d'Hypoxylon décrites depuis plus d'un siècle. Nous avons recherché les descriptions originales de ces espèces (tab. 1) en portant notre attention sur l'hôte et le lieu de la récolte. A de rares exceptions près, la matrice est une Salicacée et deux groupes d'origine géographique distincte apparaissent: Amérique boréale et Scandinavie. Nous n’avons pas observé les exsiccata relatifs aux espèces européennes et acceptons en première analyse la validité du travail de Miller. La présence d'H. mammatum serait donc ancienne et les foyers que nous décrivons en résulteraient. Les forestiers français (SILVY-LELIGEors 1949) estiment d'ailleurs qu'il existe une forte analogie entre les trembles scandinaves et ceux de la race de montagne des Alpes ou du Jura. Ces derniers seraient des reliques glaciaires des premiers. Nous manquons toutefois de critères fiables biochimiques ou génétiques (nombres chromosomiques) pour vérifier cette parenté. Curieusement, aucune mention récente de chancre n'est apparue dans la littérature scandinave.

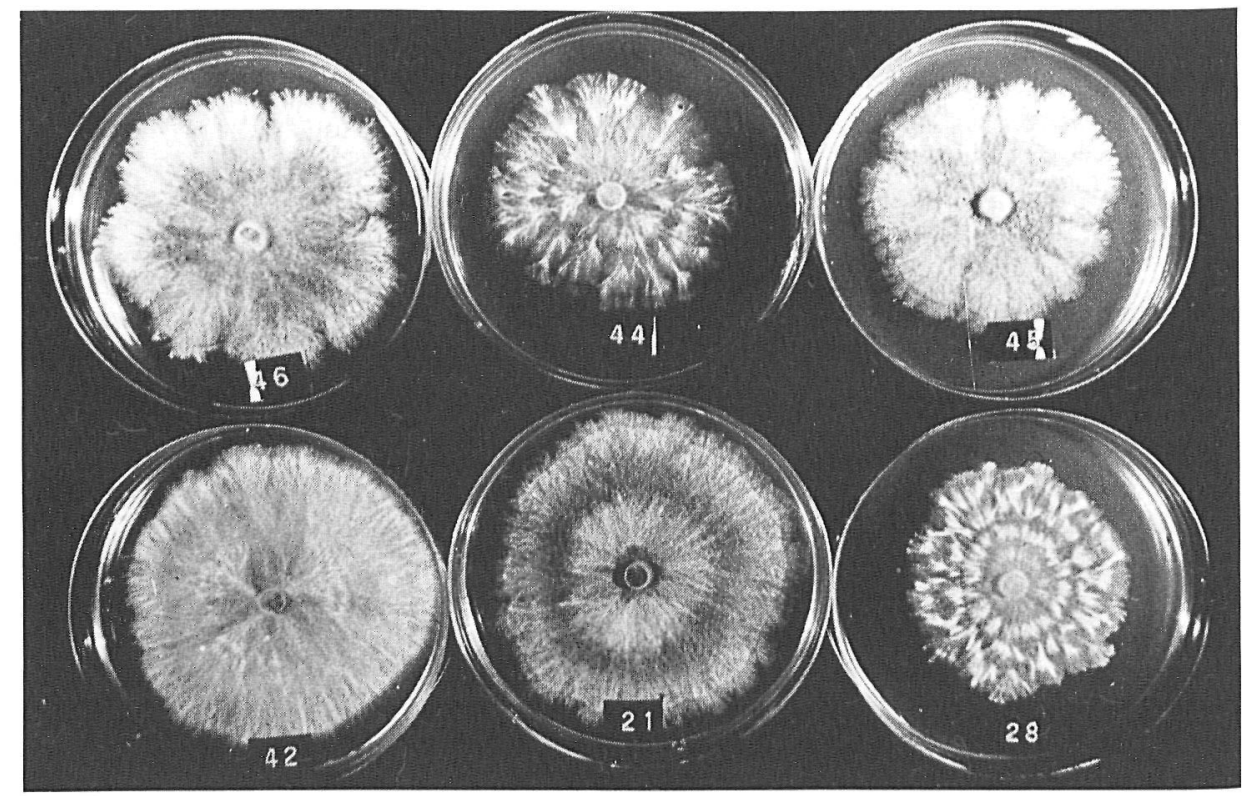

Fig. 2. Variabilité morphologique des souches d'H. mammatum in vitro 


\section{Caractéristiques culturales des souches d'H. mammatum}

Plusieurs auteurs, dont Anderson et Schipper (1975), French et Hodges (1969), French et Manion (1975) ont montré la variabilité importante du parasite in vitro selon les isolats et les matrices.

En France, la gamme d'hôtes indigènes du parasite semble limitée à $P$. tremula sous ses deux races. Ainsi, nous n'avons pu noter de chancres sur les $P$. alba de la haute vallée de la Durance, pourtant proches géographiquement des foyers alpins à haut taux d'infection. De même, aucune infection n'a été décelée à ce jour sur Bouleau.

Dès les premiers isolements, nous avons remarqué une certaine variabilité entre souches et aussi dans le temps pour une même souche. Sur 16 souches isolées en France et une au Québec, nous avons tenté d'appréhender et de caratériser au moins en partie cette variabilité (fig. 2).

Tous nos isolements proviennent de fragments de tissus prélevés en marge des chancres, là où l'isolement est le plus aisé. L'origine des souches est précisée au tableau 2. Leurs provenances géographiques et leurs matrices en sont variées.

\subsection{Aspects morphologiques}

Nos souches, à l'exception peut-être de celle d'origine québéquoise correspondent au type mycélien défini par BAGga et SMALley (1974), c'est-à-dire à croissance mycélienne

\section{Tablean 2}

Caractères qualitatifs des souches d'H. mammatum

\begin{tabular}{|c|c|c|c|c|c|c|c|c|}
\hline \multirow[b]{2}{*}{$N^{\circ}$} & \multicolumn{4}{|c|}{ Isolement } & \multirow{2}{*}{$\begin{array}{l}\text { Pigmentation } \\
\text { mout gélosé }\end{array}$} & \multicolumn{2}{|c|}{ Fructification in vitro } & \multirow{2}{*}{$\begin{array}{c}\text { Aspect } \\
\text { du } \\
\text { mycélium }\end{array}$} \\
\hline & Lieu & An. & Hote & & & $\begin{array}{l}\text { Pré- } \\
\text { cocité }\end{array}$ & $\begin{array}{c}\text { Répar- } \\
\text { tition }\end{array}$ & \\
\hline 21 & Alpes italiennes & 1975 & P.tremula & $(\mathrm{M})$ & Gris & $\mathrm{T}$ & M & M \\
\hline 22 & Chorges (A) & 1975 & P. tremula & (M) & Gris-vert & $\mathrm{T}$ & M & G \\
\hline 23 & Epinal (E) & 1975 & $\begin{array}{l}\text { P.tremula } \times \\
\text { P.tremuloides }\end{array}$ & & Gris-vert & $\mathrm{T}$ & M & M \\
\hline 24 & Les Guibertes (A) & 1975 & P.tremula & $(\mathrm{M})$ & Gris-brun & $\mathrm{P}$ & $\mathrm{E}$ & M \\
\hline 25 & Québec & 1975 & P.tremuloides & & Brun-beige & $\mathrm{P}$ & $\mathrm{E}$ & C \\
\hline 26 & St Antoine (A) & 1975 & P.tremula & $(\mathrm{M})$ & Gris verdâtre & I & $\mathrm{E}$ & G \\
\hline 27 & Clavans en Oisans (A) & 1975 & P.tremula & (M) & sans & $\mathrm{P}$ & $\mathrm{E}$ & G \\
\hline 28 & Nancy $(\mathrm{E})$ & 1975 & $\begin{array}{l}\text { P. tremula } \times \\
P . \text { tremuloides }\end{array}$ & & $\begin{array}{l}\text { gris à gris } \\
\text { verdâtre }\end{array}$ & $\mathrm{T}$ & M & M \\
\hline 37 & Le Revard (A) & 1976 & P. tremula & $(\mathrm{P})$ & $\begin{array}{l}\text { gris à gris } \\
\text { verdâtre }\end{array}$ & $\mathrm{O}$ & $\mathrm{O}$ & M \\
\hline 38 & Le Revard (A) & 1976 & P.tremula & $(\mathrm{P})$ & gris & I & $\mathrm{E}$ & M \\
\hline 39 & Plateau d'Albion (A) & 1976 & P.tremula & (M) & gris-brun & $\mathrm{P}$ & M & G \\
\hline 41 & Revest du Bion (A) & 1977 & P. tremula & (M) & gris-verdâtre & $\mathrm{T}$ & $\mathrm{E}$ & G \\
\hline 42 & Annot (A) & 1977 & P.tremula & (M) & gris-verdâtre & $\mathrm{T}$ & E & G \\
\hline 43 & Couloubrou (A) & 1977 & P.tremula & (M) & gris-verdâtre & $\mathrm{T}$ & M & G \\
\hline 44 & Crozet $(\mathrm{J})$ & 1977 & P.tremula & (M) & gris-brun & I & M & G \\
\hline 45 & Nancy (E) & 1977 & P. tremula & $(\mathrm{P})$ & brun-vert-gris & $\mathrm{P}$ & $\mathrm{E}$ & G \\
\hline 46 & Riom (MC) & 1977 & P. tremula & (?) & gris-verdâtre & $\mathrm{P}$ & $\mathrm{E}$ & G \\
\hline \multicolumn{9}{|c|}{$\begin{array}{l}\text { Lieu: (A) Alpes françaises, (E) Est, (J) Jura, (MC) Massif Central } \\
\text { Hôte : (M) race de montagne, (P) race de plaine } \\
\text { Précocité: (T) tardive, (P) précoce, (I) intermédiaire } \\
\text { Répartition : (M) marginale, (E) éparse (en boite de Pétri) } \\
\text { Aspect de la culture: M mêchue, G granuleuse, C cotonneuse }\end{array}$} \\
\hline
\end{tabular}


vigoureuse et à sporulation relativement tardive. Des distinctions entre souches existent dans la pigmentation, la relative précocité de la conidiogénèse, sa répartition sur les cultures et l'aspect du mycélium (méchu ou granuleux). Les observations relatées au tableau 2 ont été effectuées sur boîtes de Pétri alors que les cultures étaient déjà bien développées (culture à l'obscurité à $27^{\circ} \mathrm{C}$ ).

La pigmentation intervient souvent en fin de croissance avec une dominante grise mêlée plus ou moins intensément de vert ou de brun. La souche 27 en Boîte de Pétri est rapidement devenue albinos mais reprise en tube et après plusieurs mois de culture, elle présente une couleur gris-brun teintée de vert. Tous ces caractères qualitatifs manquent souvent de stabilité dans le temps, variant d'un repiquage à l'autre.

Certaines souches semblent plus aptes que d'autres à émettre par secteur des palmettes de croissance plus rapide que le reste de la culture. C'est le cas de la souche $\mathrm{n}^{\circ} 28$ à toutes les températures testées entre 11 et $31^{\circ} \mathrm{C}$. A l'inverse, les souches $21,23,26$ n'en produisent pas. Il est apparu que ce phénomène, ou son absence, était indépendant de la température ou des variations de température auxquelles nous avons soumis les cultures. Certains milieux de culture, tel le moût gélosé complémenté en grains d'avoine exacerbent la production de palmettes, alors aériennes et capables de rejoindre le couvercle des boîtes de Pétri.

\subsection{Exigences thermiques pour la croissance mycélienne}

Toutes nos cultures sont conduites sur des milieux gélosés classiques, malt à $2 \%$ ou moût de brasserie (Sylvestre et Perrin 1974). Ce dernier milieu a d'ailleurs l'avantage de procurer des cultures souvent plus régulières. L'exigence thermique a été étudiée à l'obscurité en boîtes de Pétri. La figure 3, pour la souche n ${ }^{\circ} 28$, illustre les résultats obtenus.

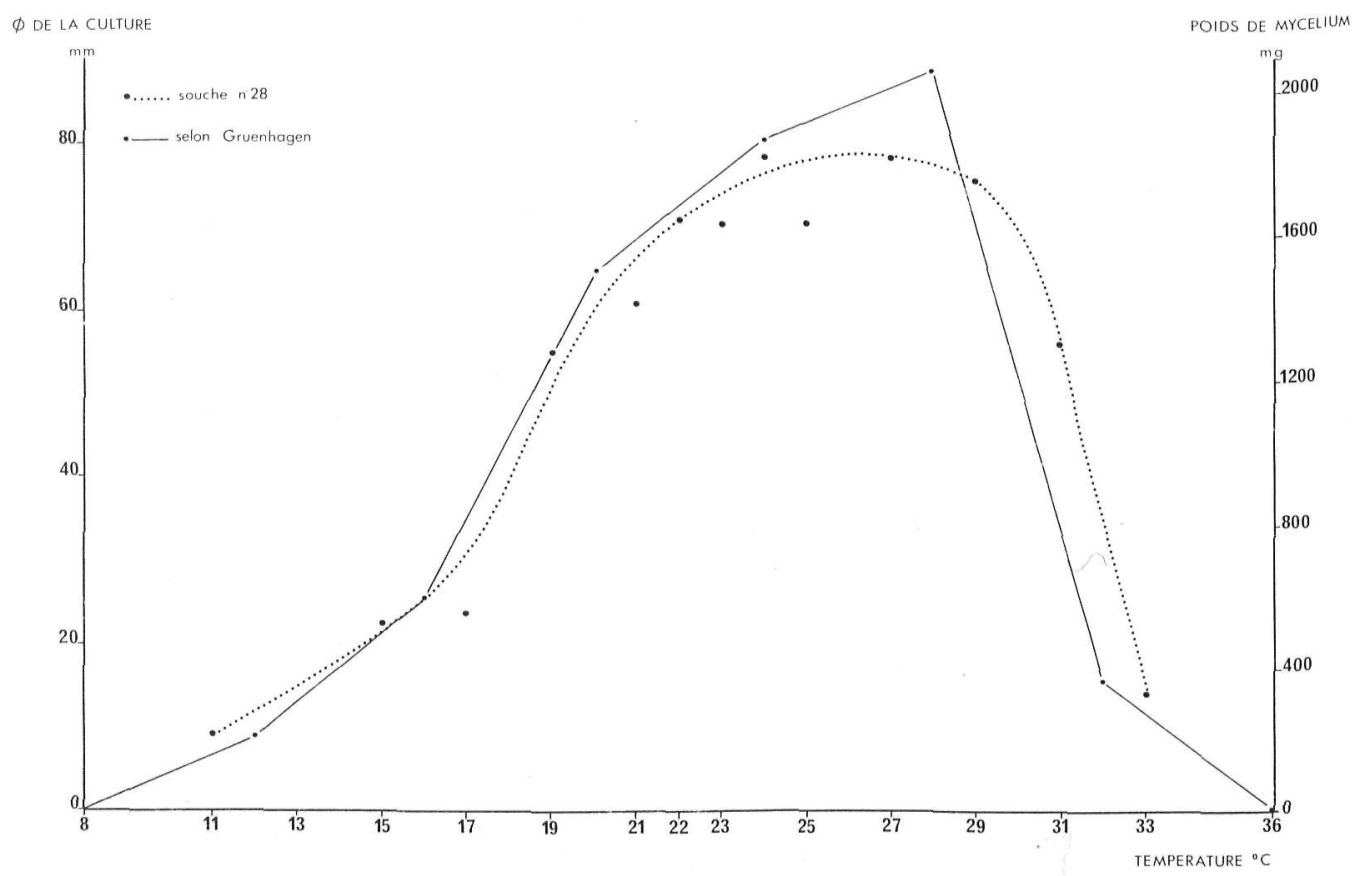

Fig. 3. Exigences thermiques : comparaison de la souche $\mathrm{n}^{\circ} 28$ (milieu gélosé et croissance radiale) aux données de GRUENHAgEN (milieu liquide et croissance pondérale) 
L'optimum de croissance se situe vers $27-28^{\circ} \mathrm{C}$. En dessous de $11^{\circ} \mathrm{C}$ et au-dessus de $32-33^{\circ} \mathrm{C}$, la croissance est pratiquement nulle. Toutes les souches testées à ce jour conduisent à des courbes de même allure qui s'accordent d'ailleurs à celles obtenues par Gruenhagen (1945) sur milieu liquide.

Selon la souche, la gamme de températures assurant la croissance la plus rapide est plus ou moins étendue, mais centrée généralement sur $27-28^{\circ} \mathrm{C}$. Les souches $\mathrm{n}^{\circ} 25,27,37,38,39$ semblent préférer $29^{\circ} \mathrm{C}$ alors que pour les souches $41,42,44$, l’optimum se situe à $25-27^{\circ} \mathrm{C}$.

Seule la souche $n^{\circ} 24$ se distingue nettement en présentant une meilleure croissance à $21-23^{\circ} \mathrm{C}$. Ni l'origine géographique ni la matrice ne peuvent expliquer ces différences.

\subsection{Vitesse de croissance}

Nous avons retenu la température de $27^{\circ} \mathrm{C}$ pour cette étude, dans la mesure où elle correspond sensiblement à l'optimum pour la grande majorité des souches. Qu'il s'agisse de cultures sur malt gélosé en boîte de Pétri ou sur milieu liquide (EghBaltalab et al. 1976) en flacons (1 mois de culture), on observe des différences importantes entre souches pour la croissance mycélienne (tab. 3). Si les deux sources de malt procurent le même classement des souches, le malt Oxoid apparaît néanmoins plus favorable. Toutefois, il n'y a pas de liaison absolue avec les résultats obtenus sur milieu liquide non agité. Ici encore l'origine des souches ne peut rendre compte des différences observées.

Tablean 3

Caractères quantitatifs des souches d'H. mammatum

\begin{tabular}{|c|c|c|c|r|r|}
\hline \multirow{2}{*}{ Souche $\left(\mathrm{n}^{\circ}\right)$} & \multicolumn{2}{c|}{ Croissance malt gélosé (mm/jours) } & \multirow{2}{*}{ Milieu liquide (mg) } & \multicolumn{2}{c|}{ Activité Endo Cx $(\%$ To) } \\
\cline { 2 - 3 } & Oxoid & Mérieux & & $3 \mathrm{~h}$ & $24 \mathrm{~h}$ \\
\hline \multirow{2}{*}{21} & 5,6 & 5,0 & 60 & 23,7 & 7,7 \\
22 & 6,1 & 4,8 & 50 & 20,1 & 7,9 \\
23 & 6,3 & 5,2 & 20 & 18,5 & 8,2 \\
24 & 2,9 & 2,3 & 50 & 34,9 & 9,5 \\
25 & 2,4 & 3,0 & 20 & 89,9 & 66,7 \\
26 & 6,1 & 5,0 & 10 & 17,5 & 6,8 \\
27 & 2,0 & 1,3 & 10 & 36,5 & 10,7 \\
28 & 5,4 & 5,2 & 20 & 22,2 & 8,6 \\
37 & 1,3 & 20 & 28,1 & 9,3 \\
38 & 5,7 & 0,7 & 30 & 16,9 & 7,6 \\
39 & 3,0 & 5,4 & 5 & 53,9 & 12,5 \\
41 & 4,9 & 1,9 & 20 & 44,5 & 12,7 \\
42 & 6,5 & 1,2 & 18,4 & 6,9 \\
43 & 6,9 & 3,1 & 20 & 14,3 & 7,1 \\
44 & 4,4 & 5,4 & 20 & 24,3 & 7,3 \\
45 & 5,2 & 4,9 & 20 & 14,9 & 7,7 \\
46 & 6,2 & 4,5 & 10 & 19,0 & 7,9 \\
\hline
\end{tabular}

\subsection{Sensibilité au Pyrocatéchol}

Hubbes (1962) et Bier (1963) ont montré que le pyrocatéchol présent dans certains tissus corticaux des faux trembles (P. tremuloides) était inhibiteur de la croissance mycélienne. Deux souches françaises ont été cultivées sur malt gélosé auquel le Pyrocatéchol commercial était ajouté à raison de 320 ou 640 ppm. Aucun développement n'est apparu à 640 ppm. A $320 \mathrm{ppm}$, la réduction de croissance varie entre 76 et $91 \%$ par rapport à la croissance des témoins. Selon Hubbes, l'inhibition est totale à 660 ppm et la croissance est limitée à $40 \%$ de celle des témoins à $330 \mathrm{ppm}$. Nos résultats sont donc du même ordre de grandeur (fig. 4). 


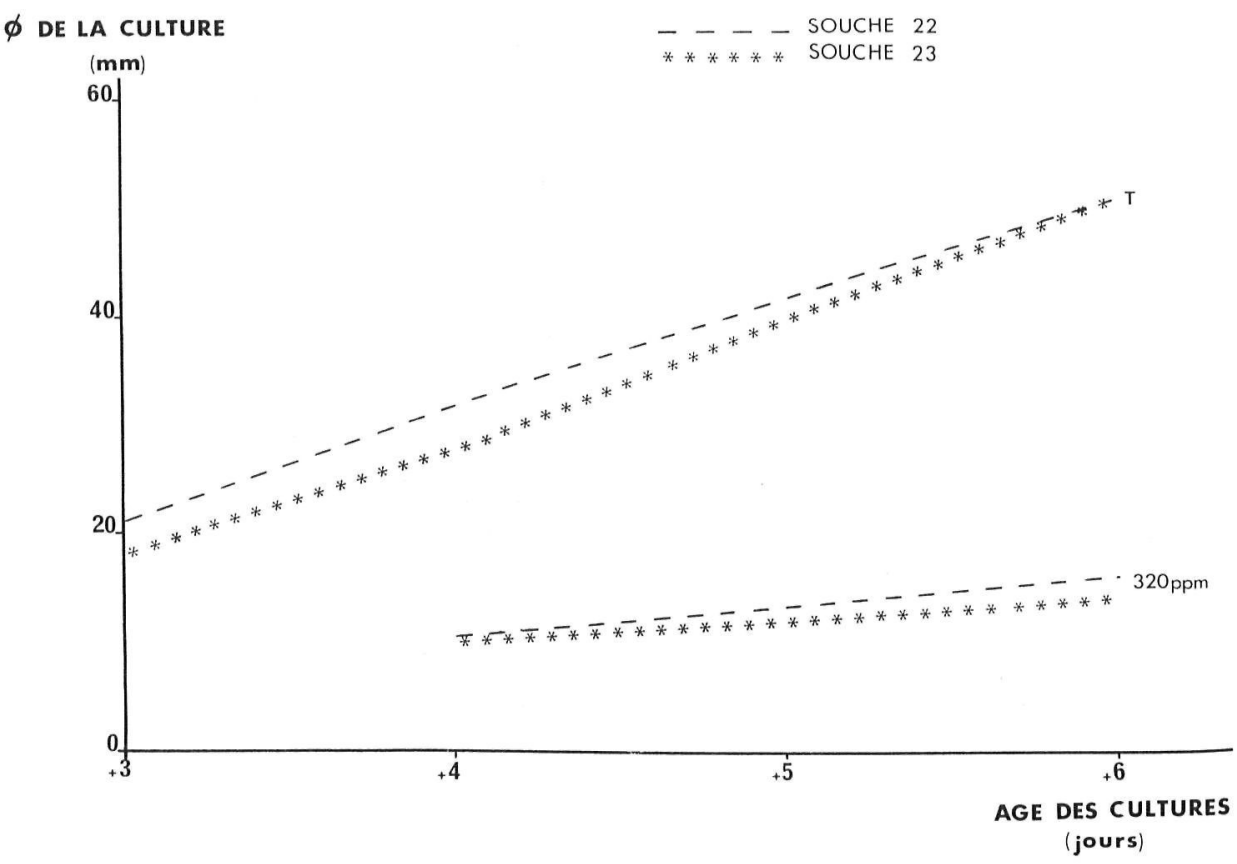

Fig. 4. Influence du pyrocatéchol sur la croissance mycélienne de souches. T $=$ milieu témoin, $320 \mathrm{ppm}$ : milieu enrichi en pyrocatéchol à 320 ppm.

\section{Pouvoir lignivore}

Les premières descriptions de symptômes se sont souvent attachées aux nécroses d'écorce ou aux chancres provoqués par H. mammatum. Hubbes (1964) a signalé ensuite le cheminement du parasite en profondeur dans le bois. BAgGa et Smalley (1969) ont insisté sur la nécessité d'atteindre le bois lors des inoculations. A de nombreuses reprises, nous avons noté que le front de progression du champignon dans le bois précédait nettement celui que laissait prévoir la limite de la décoloration corticale.

Par ailleurs, les vieux «chancres» sont des zones de faiblesse favorables aux bris par le vent. Le bois y manifeste une altération accompagnée d'une coloration de couleur anthracite. Nous avons voulu vérifier qu'H. mammatum était bien responsable de ces altérations. Nous avons retenu le test d'altération sur buchette en tubes à essais comportant un milieu gélosé incliné (moût) selon la méthodologie définie par Delatour (1975) et en étuve obscure à $27^{\circ} \mathrm{C}$.

Le tableau 4 récapitule les résultats obtenus pour deux souches d'origine géographique distincte $\left(\mathrm{n}^{\circ} 26\right.$ et $\left.\mathrm{n}^{\circ} 28\right)$ et sur deux bois de «trembles hybrides» (P. tremula $\times$ P. tremuloides et $P$. alba $\times P$. grandidentata). Aux deux durées d'expérience (152 et 388 jours) et quelle que soit la modalité envisagée, il y a perte de poids significative par rapport aux témoins. Le ralentissement du phénomène entre 152 et 388 jours peut être imputé à des difficultés expérimentales rencontrées dans le maintien à $27^{\circ} \mathrm{C}$ d'une humidité relative théorique de $75 \%$ (solution saline saturée). Le bois de P. alba $\times$ P. grandidentata a été plus altéré, phénomène qu'on ne peut pas pour autant relier à la sensibilité de chacun de ces hôtes.

Bien que les blocs soient couverts de mycélium, nous n'avons pu reproduire les aspects morphologiques d'altération connus in vivo, pas plus que la coloration. Ceci peut être 


\section{Tableau 4}

Test d'altération : évolution du poids sec en pourcentage du poids initial des épouvrettes

\begin{tabular}{|c|c|c|c|c|c|c|c|}
\hline \multicolumn{2}{|c|}{ Bois } & \multicolumn{3}{c|}{ P. tremula $\times$ P. grandidentata } & \multicolumn{3}{c|}{ P.alba $\times$ P. grandidentata } \\
\hline Souche & Durée (jours) & Min & Moy & Max & Min & Moy & Max \\
\hline \multirow{2}{*}{26} & 152 & $-1,1$ & $-7,3$ & 10,1 & $-6,5$ & $-9,7$ & $-13,2$ \\
& 388 & -4 & $-11,3$ & -16 & $-9,5$ & $-14,1$ & $-19,7$ \\
28 & 152 & $-3,2$ & $-5,6$ & $+11,0$ & $-3,4$ & $-5,8$ & -9.8 \\
& 388 & $-3,8$ & $-8,4$ & $-11,2$ & $-6,8$ & $-9,0$ & $-15,5$ \\
témoin & 152 & $+0,3$ & $+2,0$ & $+5,3$ & 0 & $+5,1$ & $+8,8$ \\
& 388 & $-1,2$ & $+1,6$ & $+4,7$ & $-0,3$ & $+3,1$ & $+8,1$ \\
\multicolumn{2}{|c}{ perte de poids, + gain de poids } & & & & & \\
\hline
\end{tabular}

attribué à l'aspect artificiel d'une méthode in vitro, ou peut signifier que d'autres microorganismes participent in vivo au phénomène. Quoi qu'il en soit, les pertes de poids sont suffisantes pour reconnaître à H. mammatum une aptitude lignivore qui explique une partie des symptômes de la maladie.

\section{Activités enzymatiques}

Les symptômes décrits sur bois ainsi que les résultats positifs des tests d'altération nous laissent à penser que l'H. mammatum dispose d'un appareil enzymatique adapté à la dégradation du bois. Nous avons donc cherché à mettre en évidence certaines activités pectinolytiques et cellulolytiques et à comparer les aptitudes respectives des différentes souches.

Dans tous les cas, nos souches ont été cultivées en étuve obscure, sans agitation à la température optimale de $27^{\circ} \mathrm{C}$.

\subsection{Recherche d'activité pectinolytique}

La souche $\mathrm{n}^{\circ} 28$ a été cultivée en erlenmeyers sur milieu liquide selon la formule d'EGHBATALAB (1976) enrichi en pectine. Diverses sources de pectine ont été offertes au champignon:

Pectine de pomme commerciale, pomme rapée ou cube de pomme émergeant du milieu, toujours à raison de $1 \%$ en poids du milieu. La recherche d'activité a été conduite uniquement sur viscosimètre d'Ostwald avec une solution tamponnée à $\mathrm{pH} 5$ (tampon de Mac Ilvaine) de pectine commerciale à $2 \%$. Pour chacune des sources de pectine, des filtrats de cultures âgées respectivement de $2-4$ et 6 semaines, 6 et 17 semaines, 4-6 et 17 semaines ont été incorporés $(1 \mathrm{ml}$ de filtrat pour $15 \mathrm{ml}$ de substrat). L'incubation a été réalisée à $30^{\circ} \mathrm{C}$ et des mesures effectuées entre 1 et 24 heures.

Cette méthode, destinée à mettre en évidence des pectine-transéliminases ou des endopolyméthylgalacturonases, n'a révélé aucune activité, c'est-à-dire, aucune réduction notable de la viscosité du milieu en incubation.

\subsection{Activité cellulolytique}

Nos essais ont porté sur la détection par viscosimétrie de l'activité de type endo Cx selon la méthode décrite par KeLman et CowLING (1967) et sauf mention contraire sur la souche $\mathrm{n}^{\circ} 28$ cultivée à l'obscurité à $27^{\circ} \mathrm{C}$ sur milieu liquide enrichi de cellulose. L'incubation a toujours porté sur un mélange de $15 \mathrm{ml}$ de sel sodique de carboxyméthyl-cellulose à 1,2\% tamponné à pH 5,5 et de $1 \mathrm{ml}$ defiltrat de culture. Les tubes viscosimétriques étaient plongés 


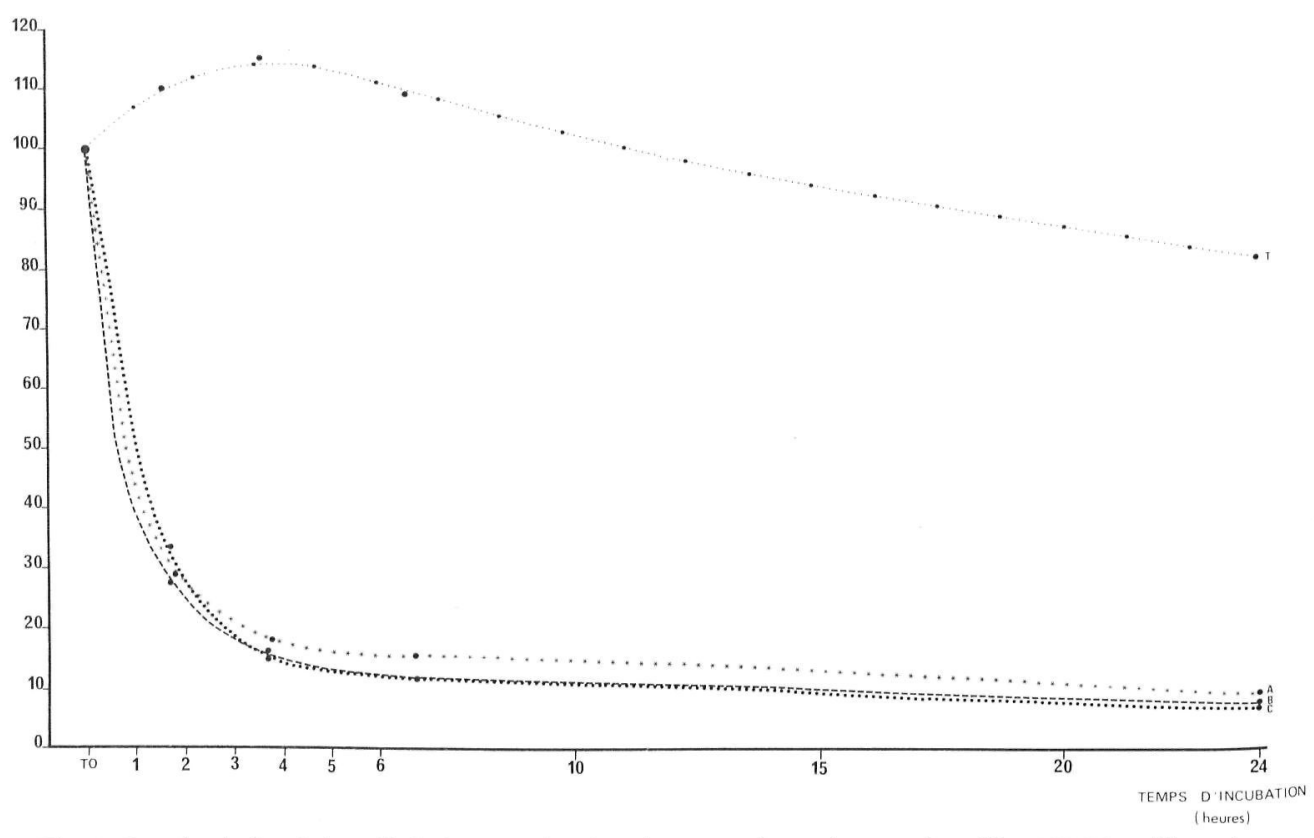

Fig. 5. Courbe de l'activité cellulasique : réduction du temps d'écoulement du milieu (CMC + filtrat de culture) pendant l'incubation à $30^{\circ} \mathrm{C}$, de 0 à 24 heures. $\mathrm{T}=$ témoin, $\mathrm{A}, \mathrm{B}, \mathrm{C}=3$ répétitions avec filtrat de culture

dans un bain thermostaté agité réglé à $30^{\circ} \mathrm{C}$ et les mesures du temps d'écoulement pratiquées jusqu’à 24 heures.

Dans ce qui suit et pour plus de commodité, nous conviendrons d'appeler «to» le moment de mise en contact du substrat et du filtrat et de rapporter en heures à partir de cet instant les temps de lecture. La durée moyenne d'écoulement à to est systématiquement affectée de l'indice 100 et les durées ultérieures exprimées en pourcentage par rapport à cette valeur. Nous les appellerons écoulements relatifs (par rapport à l'instant initial) (fig. 5). Chaque modalité a fait l'objet d'au moins trois répétitions et jusqu'à 10 dans le cas de la comparaison des souches. Dans les témoins, de l'eau permutée remplace en proportions identiques le filtrat de culture. Pour chaque essai, il a été vérifié que l'ébullition des filtrats annulait l'essentiel de leur activité qui pouvait donc être attribuée à une molécule thermolabile, en pratique une enzyme ou un mélange d'enzymes. Nos premiers essais présentent un caractère méthodologique: choix du milieu de culture, du substrat cellulosé, de l’âge des cultures.

\subsubsection{Choix d'un milieu de culture}

Deux milieux de culture liquides, celui d'Eghbaltalab et un milieu de Richard pauvre $\left(\mathrm{KNO}_{3} 10 \mathrm{~g} / 1, \mathrm{KH}_{2} \mathrm{PO}_{4} 5 \mathrm{~g} / \mathrm{l}, \mathrm{MgSO}_{4} 7 \mathrm{H}_{2} \mathrm{O} 2,5 \mathrm{~g} / \mathrm{l}, \mathrm{FeCl}_{3} \mathrm{O}, 02 \mathrm{~g} / \mathrm{l}\right)$ enrichis en diverses sources de glucide ou de cellulose ont été éprouvés après 45 jours de cultures dans les conditions déjà décrites pour leur aptitude à induire l'activité cellulolytique. Le tableau 5 résume l'essentiel des données recueillies avec la souche $\mathrm{n}^{\circ} 28$.

En l'absence de cellulose, aucune activité n'est décelable, même à l'issue de 24 heures d'incubation. La cellulase recherchée ne peut donc être qu'adaptative. Le résultat est aussi négatif si on ajoute une source sucrée à la cellulose. Les deux milieux s'avèrent favorables, celui d'Eghbaltalab fournissant toutefois un développement mycélien plus intense. 
Enfin, sur Richard pauvre, nous avons testé l'efficacité de diverses sources de cellulose à raison de $1 \mathrm{~g}$ par erlenmeyer contenant $100 \mathrm{mldemilieu}$ liquide: sel sodique de C.M. C., papier, coton, pâte de bois de tremble. Leur pouvoir inducteur est fort différent, l'activité étant respectivement de $2 \%, 78 \%$ et $55 \%$ de celle obtenus avec la pâte de bois, prise comme base 100 .

Nous avons donc retenu pour la suite de notre étude le milieu d'Eghbaltalab complémenté en pâte de bois à raison de $1 \%$ en poids.

\section{Tablean 5}

Activité cellulolytique in vitro selon la nature du milieu de culture à 45 jours

\begin{tabular}{|c|l|c|}
\hline Milieu de base & Source de carbonne & $\begin{array}{c}\text { Réduction du temps } \\
\text { d'écoulement }\end{array}$ \\
\hline Richard & Nulle & 0 \\
& $\begin{array}{l}\text { Papier }(10 \mathrm{~g} / 1) \\
\text { Papier }+ \text { sucrose } \\
\text { Eghbaltalab }\end{array}$ & $45 \%$ \\
& $\begin{array}{c}\text { Sucrose } / 1) \\
\text { Nulle }\end{array}$ & 0 \\
& $\begin{array}{c}\text { Papier }(10 \mathrm{~g} / 1) \\
\text { Pâte de tremble } \\
(10 \mathrm{~g} / 1)\end{array}$ & 0 \\
& & $38 \%$ \\
\hline
\end{tabular}

\subsubsection{Effet de l'âge des cultures sur l'activité cellulolytique}

Sous les conditions culturales déjà définies et avec quatre répétitions, nous avons étudié le développement progressif de l'activité cellulolytique selon l'âge des cultures. La figure $\mathrm{n}^{\circ} 6$ illustre les résultats obtenus, pour une durée de 3 heures d'incubation. Le temps d'écoulement diminue régulièrement entre la lère et la 4è semaine de culture. Elle atteint alors son minimum avec une moindre dispersion des valeurs entre répétitions. Au-delà, l'activité persiste à un niveau pratiquement constant. Les cultures âgées d'un mois conviennent donc et seront seules utilisées par la suite.

\subsubsection{Activité cellulolytique des diverses souches}

Hypoxylon mammatum est donc capable de développer in vitro une activité endo Cx. Toutefois, la variabilité décrite pour d'autres caractères nous a conduit à comparer nos souches pour leur activité. Le tableau 3 montre qu'après 3 heures d'incubation, des différences

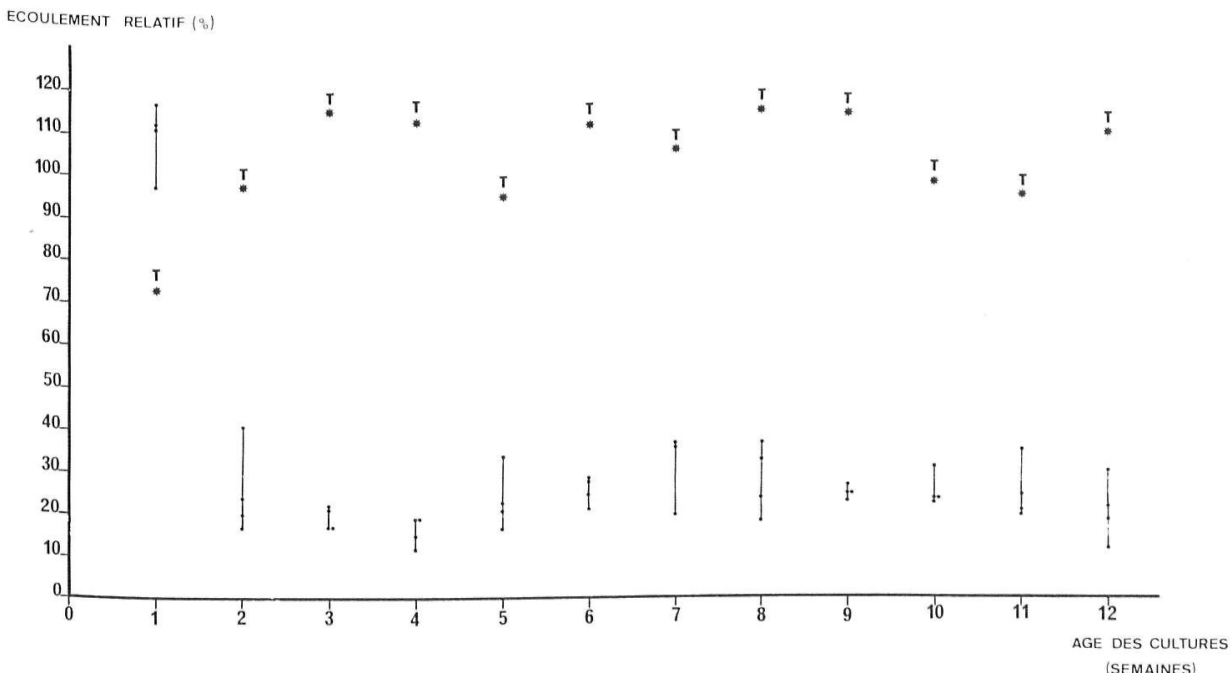

Fig. 6. Développement de l'activité cellulolytique selon l'âge des cultures : temps moyen d'écoulement du milieu (CMC + filtrat de culture) après 3 heures d'incubation à $30^{\circ} \mathrm{C}$ 
notables apparaissent entre les souches. La souche 39 et surtout la souche 25 sont peu actives, les souches $24,27,37$ et 41 le sont nettement et enfin toutes les autres sont très actives. Ni l'hôte, ni la date, ni le lieu d'isolement ne peuvent rendre compte des comportements observés. Bien que le nombre de souches étudiées soit modeste, il semble que celles manifestant une croissance rapide sur milieu gélosé sont aussi celles qui présentent les plus fortes activités. Un test de même nature réalisé après 45 jours de culture a confirmé les différences entre souches. Dans la mesure où l'activité s'exprime totalement dans les 4 premières semaines de culture, on peut penser que l'activité cellulolytique reflète plus une vigueur des souches que la nécessité d'atteindre un niveau minimal de développement mycélien. Néanmoins, pour des vitesses de croissance comparables, des différences non négligeables d'activité distinguent nos souches.

On notera qu'au cours de cet essai, nous avons été amenés à substituer la pâte d'un peuplier euraméricain (clone I-214) à la pâte de tremble sans que cela affecte l'induction de la cellulase fongique.

\section{Conclusions}

L'étude de la répartition géographique des foyers actuels, les observations de Messiaen relatives au Sud des Alpes, l'analyse (bibliographique) des mentions relatives aux espèces réduites en synonymie par Miller, tendent à démontrer qu'Hypoxylon mammatum est un parasite qui sévit de longue date en Europe. Le peu d'intérêt porté aux trembles peut expliquer le caractère récent des mentions faites par plusieurs pathologistes d'Europe (Tchécoslovaquie, France, URSS), à moins que des modifications climatiques récentes aient pu en renforcer les caractères visibles.

L'impact du parasite est sans conteste plus sérieux en montagne et pour l'instant seules des hypothèses peuvent être avancées. Génétiquement, le tremble de la race de montagne est distinct de celui de plaine. Des inoculations seront conduites pour déterminer si la première race est plus réceptive. Au sein de la zone alpine, le pourcentage d'arbres attaqués est nettement plus élevé dans le Sud de l'aire, où l'influence du climat méditerranéen est marquée. BAGGA et SMALLEY (1967) ont montré qu'un stress hydrique favorise l'installation du parasite. Des conditions de stress sont mieux réalisées en zone alpine où le tremble croît sur des pentes à sols superficiels et à fortiori dans le Sud où les précipitations estivales sont plus rares.

La variabilité décrite à propos de nos souches, leurs exigences thermiques, leurs caractères qualitatifs confirment les études nord-américaines. Le pouvoir lignivore, qui pour partie traduit l'activité cellulolytique, est un fait nouveau. La variabilité notée à ce propos pourrait expliquer des différences d'agressivité décrites par les chercheurs américains. Ceuxci ont noté en effet des différences dans la vitesse d'extension des chancres. Si l'activité cellulolytique mise en évidence in vitro est mise à profit in vivo par le parasite pour coloniser de nouveaux tissus ligneux, elle pourrait rendre compte de ces différences d'agressivité. Une étude est en cours pour vérifier cette hypothèse.

Outre son intérêt fondamental, cet aspect doit être pris en compte pour le choix des souches destinées aux tests de sensibilité.

\section{Remerciements}

Nos remerciements s'adressent à Mme Arlette Schipfer pour son assistance technique. Nous y associons Mme Gilberte Guinot et M. Janin qui ont facilité nos études sur l'altération et la cellulolyse ainsi que MM. Delatour, Messiaen et Perrin qui nous ont communiqué des localisations du parasite. 


\section{Résumé}

Hypoxylum mammatum, l'agent du «chancre» du tronc des trembles est largement répandu en France. Il est particulièrement fréquent et actif sur les peuplements de montagne (Alpes, Jura), mais plus rare et plus discret dans les peuplements de plaine.

Son origine est discutée. Il est acquis que la maladie sévit depuis 25 ans dans les Alpes et il est probable que son existence en Europe date du siècle dernier.

Les souches isolées en France présentent une grande variabilité morphologique in vitro et des exigences thermiques pour la croissance comparables à celles des souches nord-américaines. Une partie des symptômes semble le fait du pouvoir lignivore et de l'activité cellulolytique du type Endo Cx mis en évidence au cours de l'étude. Ces caractères s'avèrent variables en intensité selon les souches et leur liaison éventuelle avec l'agressivité sera ultérieurement approfondie.

\section{Summary}

Hypoxylon mammatum, causing stem canker of aspen is widely distributed in France. Frequent and damaging in mountain stands, it appears to be relatively rare and localised in the plains.

Its origin is discussed. The disease has probably been present in the Alps for the last 25 years and perhaps in Europe since last century.

French isolates appear very variable in their in vitro morphological characters and resemble American isolates in their temperature-growth relationships. Part of the symptoms may be attributed to the wood decomposing ability of the fungus and to its production of Endo Cx type of cellulase. These features are variable among isolates and their role in aggression will be further explored.

\section{Zusammenfassung}

Ursprung und Hauptmerkmale französischer Hypoxylon mammatum-Isolate

Hypoxylon mammatum, Erreger eines Stammkrebses an Aspe, ist in Frankreich weit verbreitet. Häufig und schädlich in Bergwäldern, scheint er im Flachland nur relativ selten und örtlich begrenzt vorzukommen.

Der Ursprung des Pilzes wird diskutiert. Wahrscheinlich kommt die Krankheit während der letzten 25 Jahre in den Alpen vor und ist in Europa seit dem letzten Jahrhundert vertreten.

Französische Isolate erscheinen in den morphologischen in vitro-Merkmalen sehr variabel, und sie ähneln amerikanischen Isolaten in ihrem temperaturbedingten Wachstumverhalten. Ein Teil der Symptome könnte mit dem Holzabbauvermögen des Pilzes und mit seiner Produktion des Endo-Cx-Typs der Cellulase zusammenhängen. Diese Eigenschaften schwanken zwischen den Isolaten; ihre Bedeutung für die Pathogenität wird weiter untersucht.

\section{Bibliographie}

Anderson, G. W.; Schipper JR., A. L., 1975: Variation among isolates of Hypoxylon mammatum. Eur. J. For. Path. 5, 216-224.

Bagga, D. K.; Smalley, E. G., 1967: Water stress in relation to initiation and development of Hypoxylon canker of aspen. Phytopath. 57, 802 (Abstr.).

- 1969: Factors affecting canker development on Populus tremuloides artificially inoculated with Hypoxylon pruinatum. Can. J. Bot. 47, 907-914.

- 1974: Variation of Hypoxylon pruinatum in cultural morphology and virulence. Phytopath. 64, 663-667.

BIER, J. E., 1963: Some inoculum and substrate factors in the cultural inhibition of Hypoxylon pruinatum (Klotzsche) Cke. by Pyrocatechol. Can. J. Bot. 41, 1585-1596.

Bouvarel, P.; Fourchy, P., 1954: A propos du tremble des Alpes, les trembles de la vallée du Ferrand (Isère). Rev. For. Fr. 10, 601-604.

Delatour, C., 1975: Comportement in vitro du Sparassis crispa Wulf. ex Fr. et du Sparassis laminosa Fr. Eur. J. For. Path. 5, 240-247.

Eghbaltalab, M.; Debaud, J. C.; Bruchet, G., 1976: Etude in vitro des activités pectinolytique et cellulolytique du Fomes annosus (Fr.) Cke et de Rosellinia quercina. Bull. mens. Soc. Linn. Lyon $45,85-90$.

French, D. W.; Hodges, C. S.; Froyds, J. D., 1969: Pathogenicity and taxonomy of Hypoxylon mammatum. Can. J. Bot. 47, 224-226. 
French, J. R.; Manion, P. D., 1975: Variability of host and pathogen in Hypoxylon canker of aspen. Can. J. Bot. 53, 2740-2744.

Gruenhagen, R. H., 1945: Hypoxylon pruinatum and its pathogenesis on poplar. Phytopath. 35, 72-89.

Hubbes, M., 1962: Inhibition of Hypoxylon pruinatum by Pyrocatechol isolated from bark of aspen. Sci. 136, 156.

- 1964: Host parasite relationship in the Hypoxylon canker of aspen. Breeding pest resistant trees. Oxford: Pergamon Press.

Kelman, A.; Cowling, E. B., 1967: Measurement of Cellulase activity of plant pathogens using a viscosimetric technique. Source book of Laboratory exercises in Plant Pathology. San Francisco and London: Freeman and Comp.

Kalandra, A., 1966: Rakovina topolu, doprovazena houbou z rodu Hypoxylon V. C.S.S.R. Lesn. Cas. 12, 487-492.

Lemoine, M., 1973: Amélioration des peupliers de la section Leuce sur sols hydromorphes. Thèse Université de Nancy I, 120 pp.

Miller, J. M., 1961: A monograph of the world species of Hypoxylon. Un. of Georgia Press, 158 pp. Pinon, J., 1975: Prśence en France du «chancre» du tremble Hypoxylon mammatum (Wahl.) Mill. C. R. Acad. Agric. Fr. 61, 703-706.

Schipper JR, A. L., 1975: Hypoxylon pathotoxin necessary to the infection of aspen by Hypoxylon mammatum. Proc. Amer. Phytopath. Soc. 2, 46.

Shirnina, L. V.; Kireeva, S. V., 1976: Variabilité des caractéristiques morphologiques et culturales d'Hypoxylon pruinatum. Mikol. i Fitopatol. S.S.S.R. 10, 143-146.

Silvy-Leligeois, P., 1949: Observations sur le tremble en Maurienne. Rev. For. Fr. 8, 362-370.

Sylvestre, G.; Perrin, R., 1974: Retour aux vieilles méthodes, utilisation du mout de brasserie dans la fabrication des milicux, 4 pp., Doc. ${ }^{0} 74-3$ Lab. Path. For., Nancy.

Urosevic, B., 1972: The occurrence of Hypoxylon canker of poplar in Czechoslovakia. Pr. Vyzk. Ust. Les. Hosp. Mysh. 42, 119-128.

Adresse de l'autenr: J. Pinon, Laboratoire de Pathologie forestière, I. N.R.A., Champenoux, F-54280 Seichamps

\title{
Influence of zinc smelter emissions on peroxidase activity in Scots pine needles of various families
}

\author{
By ST. Niemtur
}

Receipt of Ms. 23. 5. 1978

\begin{abstract}
Great differences in peroxidase activity were found in pine needles of 58 families, growing in two experimental areas at differing distances from the Zinc Metallurgical Works. Such differences reduce the diagnostic value of p.a., and may be important in the selection of Scots pine.
\end{abstract}

\section{Introduction}

The influence of different components of industrial pollution on peroxidase activity (p.a.) has been studied since 1962 (DëssLer 1962). Many reports appeared in the seventies (KELLER and Schwager 1971; Keller 1974; Keller et al. 1976; Keller 1977). Interest was mainly focussed on the influence of different toxic compounds on p.a. in leaves of particular 
This document is a scanned copy of a printed document. No warranty is given about the accuracy of the copy. Users should refer to the original published version of the material. 